\title{
Infrared thermal imaging in connective tissue diseases
}

\section{Marek Chojnowski}

Department of Connective Tissue Diseases, National Institute of Geriatrics, Rheumatology and Rehabilitation, Warsaw, Poland

\begin{abstract}
Infrared thermal imaging (IRT) is a non-invasive, non-contact technique which allows one to measure and visualize infrared radiation. In medicine, thermal imaging has been used for more than 50 years in various clinical settings, including Raynaud's phenomenon and systemic sclerosis. Imaging and quantification of surface body temperature provides an indirect measure of the microcirculation's overall performance. As such, IRT is capable of confirming the diagnosis of Raynaud's phenomenon, and, with additional cold or heat challenge, of differentiating between the primary and secondary condition. In systemic sclerosis IRT has a potential role in assessing disease activity and monitoring treatment response. Despite certain limitations, thermal imaging can find a place in clinical practice, and with the introduction of small, low-cost infrared cameras, possibly become a part of routine rheumatological evaluation.
\end{abstract}

Key words: Raynaud's phenomenon, systemic sclerosis, thermography, thermal imaging.

\section{Introduction}

Infrared thermal imaging (infrared thermography, IRT) is a non-invasive technique which allows one to measure and visualize infrared (IR) radiation. Prototype IR devices were developed by the military during and after the Second World War. In the late 1950s, IR technology was made available for industry and civilian science. At the turn of the century, thanks to steady technological progress, the bulky IR cameras with liquid nitrogen cooling were replaced by portable, commercially available devices. This led to more widespread use of IRT in diverse fields of science, including medicine.

This review will explore the current and possible future applications of IRT in diagnostics of Raynaud's phenomenon and related connective tissue diseases.

\section{Physical and physiological principles}

Infared radiation is a part of the electromagnetic spectrum, covering the range of wavelengths longer than visible light between $700 \mathrm{~nm}$ and $1 \mathrm{~mm}$ [1]. According to the Stefan-Boltzmann law, the amount of IR radiation emitted by a surface of any given object is pro- portional to the fourth power of the object's thermodynamic temperature (expressed in degrees Kelvin) [2]. In other words, the warmer the object, the more thermal radiation it emits. IRT allows visualization and quantification of this phenomenon, providing information on the temperature range of the observed object.

The average human body core temperature is approximately $37 \pm 0.5^{\circ} \mathrm{C}$, with surface temperature slightly lower and more variable, depending on ambient conditions [3]. Several pathological processes can induce either systemic or local thermal anomalies, e.g. increased temperature due to infection, inflammation, trauma and malignancy or decreased temperature due to ischemia. Since physiological alterations, such as temperature change, precede anatomical abnormalities observed in classical medical imaging, IRT is a potential tool for very early detection of these conditions.

It should be stressed, however, that multiple factors such as age, time of day and year, state of consciousness, emotions, activity level, hormonal imbalance and medications can have a great influence on human body temperature [4]. Accordingly, thermograms should be interpreted with caution, in the context of the full clinical picture and results of other imaging studies. 


\section{Technical considerations and patient preparation}

Since numerous external factors may negatively influence the results of IRT, both the study room and the patient should be appropriately prepared [5]. The ambient room temperature should be stable between $18^{\circ} \mathrm{C}$ and $25^{\circ} \mathrm{C}$, optimally $22-24^{\circ} \mathrm{C}$. If it is too cold, the autonomic reflex will cause vasoconstriction, and too much heat may cause excessive sweating, both of which alter the study outcome.

Before the examination, the patient should be acclimatized to the study room temperature for at least 15 minutes. For 4-6 hours before IRT, the patient should avoid smoking, drinking alcohol and excessive amounts of coffee or tea, eating large meals, using cosmetics and ointments on the examined area and performing strenuous physical activity, including all kinds of physiotherapy (e.g. massage or cryotherapy). The use of medications influencing the vascular system, such as beta-blockers or nitrates, should be reported to the performing physician before the study.

It should be noted that IRT is an absolutely safe, non-contact imaging modality, which presents no danger or radiation exposure for either the patient or the performing physician. The image acquisition itself is very fast, and, if necessary, can be repeated at short time intervals.

\section{Clinical applications of IRT}

In medicine, IRT has been used for more than 50 years. The first clinical experiments were conducted in the field of oncology, mainly for diagnosis of breast cancer and malignant melanoma. They were followed by attempts to study therapy response in inflammatory arthritis, visualize musculoskeletal injuries, locate tender points in fibromyalgia, diagnose complex regional pain syndrome (algodystrophy), monitor wound healing after surgery and evaluate microcirculation in vascular diseases. During the epidemic of SARS (severe acute respiratory syndrome) in South East Asia, IRT was used for passenger fever screening at the airports [6].

Currently, despite ongoing research, the role of IRT in most clinical settings is equivocal, mainly due to the absence of standardized study protocols and relatively low availability of thermal imaging equipment.

\section{Raynaud's phenomenon}

Raynaud's phenomenon (RP) is an episodic, transient ischemia in response to cold or emotional stimuli [7]. In most of the patients its character is benign, with no known cause or underlying condition (primary
RP, pRP). However, in a small percentage of cases, RP is the first symptom of a connective tissue disease, most commonly systemic sclerosis (SSc) or another scleroderma-spectrum disorder (secondary RP, SRP). The differentiation between pRP and SRP is of paramount importance. Primary RP requires only a conservative approach, whereas diagnosis of RP secondary to connective tissue disease mandates further diagnostics and treatment.

The most important tool for differential diagnosis of primary and secondary RP is nailfold videocapillaroscopy (NVC), which permits direct visualization of the blood vessel structure [8]. However, it provides little or no information about the nature of the blood flow. Moreover, NVC does not allow for actually diagnosing RP, since the capillary image in PRP and a healthy control is essentially the same (i.e. normal). Accordingly, initial diagnosis of $\mathrm{RP}$ is usually based only on the clinical presentation and the patient's history [9].

These limitations can be overcome with functional imaging modalities, including IRT. In the trunk and proximal body parts, the surface temperature is heavily influenced by internal organs, muscle activity and subcutaneous tissue. In contrast, in the most acral parts, such as hands and fingers, the surface temperature is almost exclusively regulated by constriction and dilation of blood vessels. As such, skin temperature recorded with IRT provides an indirect measure of microcirculation's overall performance.

Several studies report that IRT can help detect RP [10-12]. In healthy subjects, the fingers, especially the finger tips, are usually warmer than the hand. In RP patients the fingers are significantly colder than the hands, even between ischemic episodes. Lim et al. found that a temperature difference of $2.2^{\circ} \mathrm{C}$ between the fingers and the hand (in favor of the hand) is indicative of RP [13]. Studies by Murray et al. [14] and Schlager et al. [15] concluded that thermal abnormalities in RP patients detected with IRT have a good correlation with actual skin perfusion, determined by laser Doppler perfusion imaging (LDPI). Both authors also suggest that these two techniques may be used interchangeably.

To further differentiate between PRP and SRP, IRT is performed at baseline and after some type of temperature challenge. Cold challenge is the most commonly used. After immersion of the hands in cold water, rewarming time is measured. In pRP patients, rewarming is slightly delayed compared to unaffected subjects, while in SRP the hands remain cold for a prolonged time. Some authors, however, question the value of cold challenge, suggesting that baseline thermograms have enough discriminatory power for RP diagnosis [10, 15].

Instead, it has been proposed that a heat challenge can be used for RP diagnosis [11]. The increase in tem- 

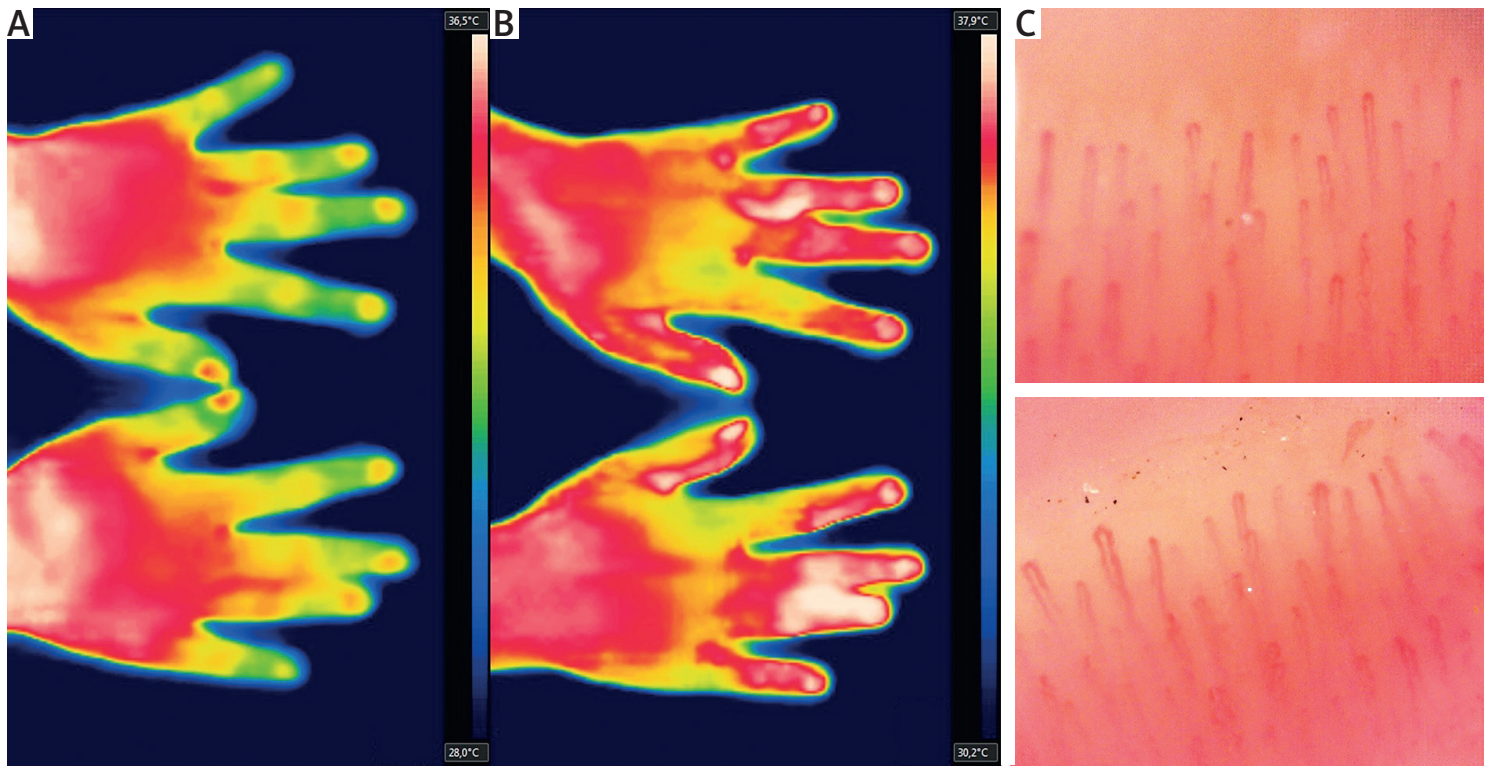

Fig. 1. Primary Raynaud's phenomenon at baseline (A), with symmetrical finger rewarming after heat challenge (B). Normal capillaroscopic image is consistent with primary nature of the condition (C).
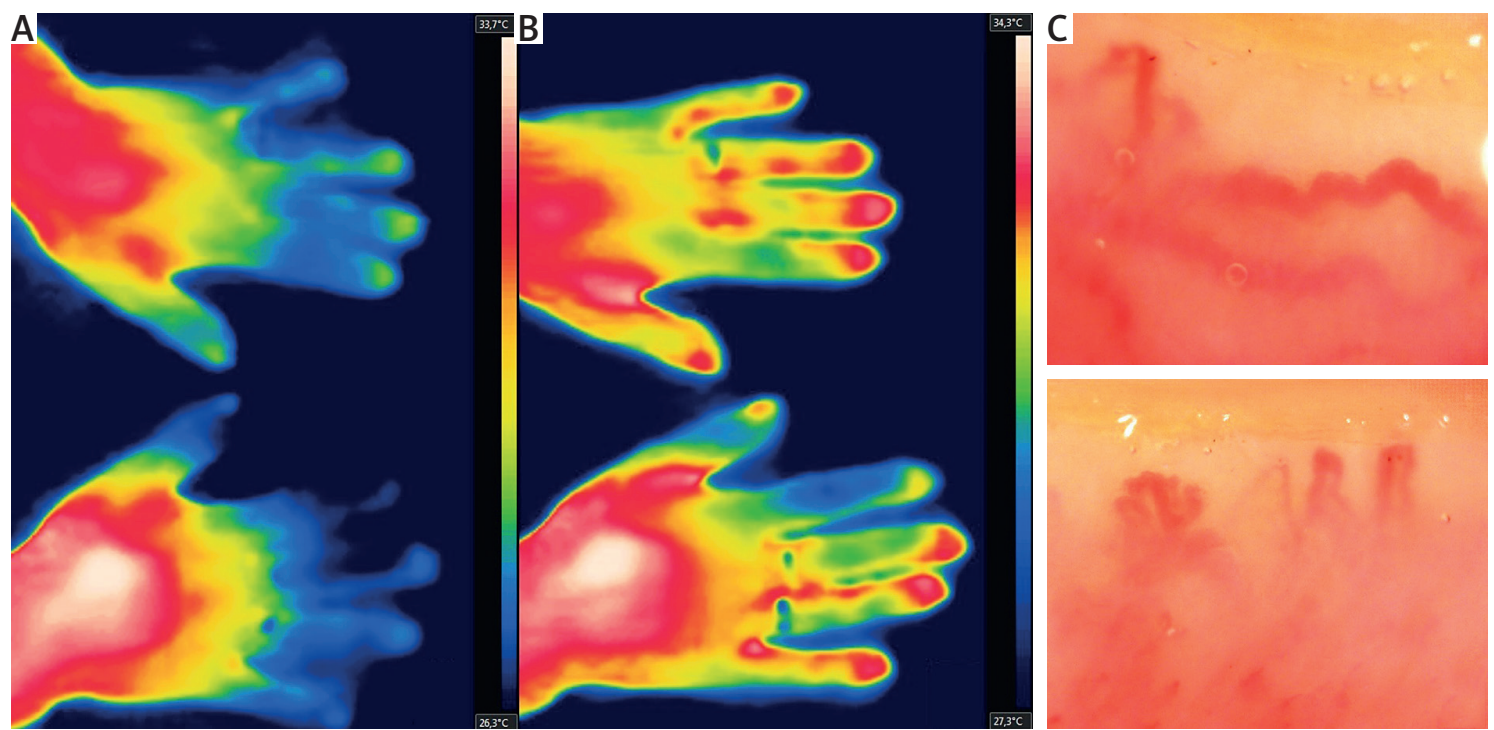

Fig. 2. Raynaud's phenomenon secondary to dermatomyositis at baseline (A), with only partial rewarming after heat challenge (B). Giant-ramified capillaries are suggestive of dermatomyositis-pattern (C) [24].

perature forces vasodilation, returning the normal thermal pattern in pRP patients (fingers warmer than the hand) (Fig. 1). In contrast, the vasodilatory response in SRP (e.g. in SSc) raises finger temperature only marginally, since restriction of blood flow is caused mainly by irreversible, structural vessel damage, not vasospasm (Fig. 2, 3).

When performing the temperature challenge, one should also take into consideration the patients' com- fort. In most of the RP subjects, a rapid decrease in temperature may trigger vasospasm, almost universally associated with unpleasant sensations, such as numbness, pain or burning. In contrast, the heat challenge does not trigger RP, and as such is better tolerated.

While the role of IRT as a single study in RP diagnostics remains ambiguous, it is a powerful complement to NVC. Combining information about blood vessel structure from NVC with functional evaluation of microcircu- 

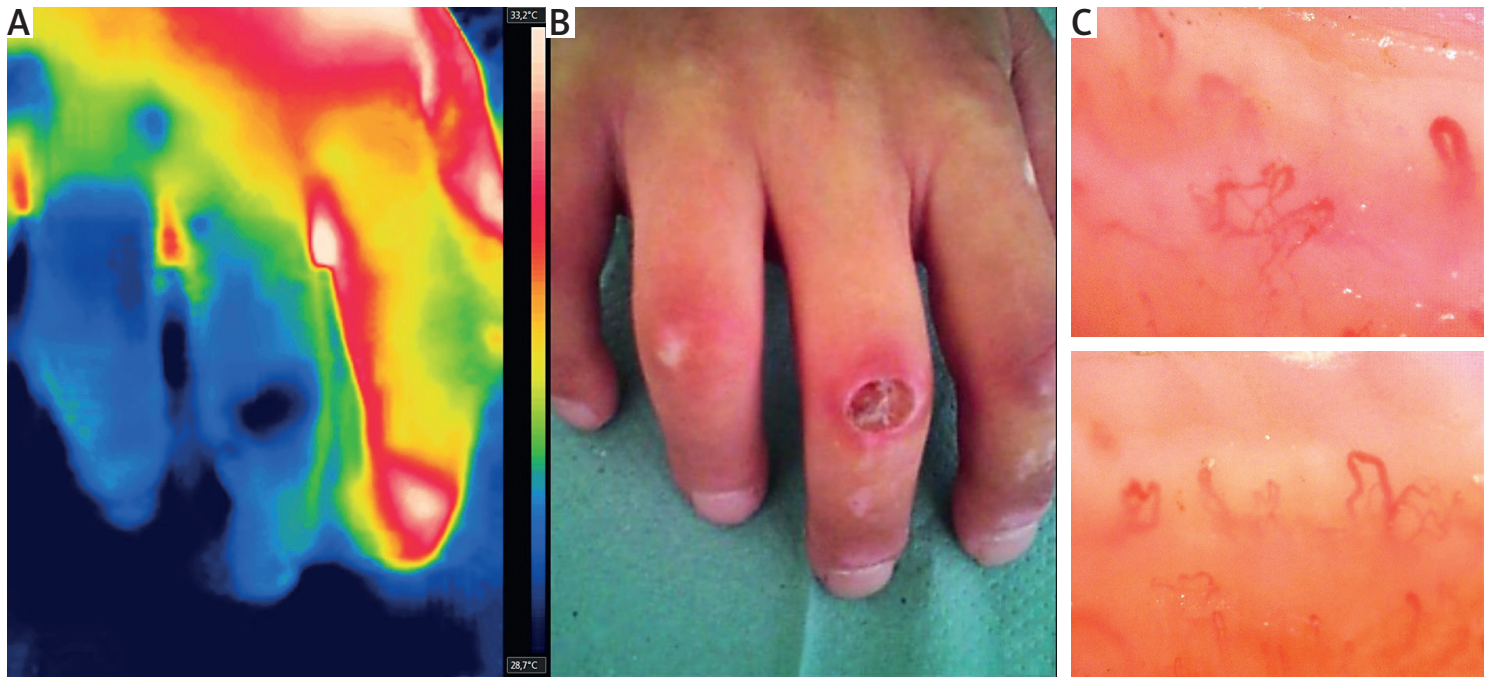

Fig. 3. Thermographic (A) and clinical (B) presentation of digital ulcer in systemic sclerosis. Capillaroscopy consistent with 'late' scleroderma-pattern (C).

lation was proven to have better specificity and sensitivity in RP differential diagnosis than NVC alone $[14,16]$.

\section{Systemic sclerosis and scleroderma- spectrum disorders}

Systemic sclerosis is an autoimmune disease characterized by progressive dysfunction of the microcirculation and tissue fibrosis [17]. Contrary to pRP patients, in SSc severe episodes of RP are a manifestation of ongoing vascular damage, which may lead to formation of non-healing digital ulcers (DUs) and subsequent tissue necrosis.

As mentioned above, IRT may play an important role in the initial diagnosis of SSc. However, its significance in assessing disease activity or monitoring treatment response is less clear. One of the promising clinical applications is determining the status and nature of DUs. A focal temperature increase of the DU, in otherwise hypoperfused fingers, is strongly suggestive of active inflammation. In parallel, a 'cold spot' might indicate ischemia and presence of dead tissue (Fig. 3). Weijden et al. [18] reported an interesting case of an SSc patient in whom IRT made it possible to differentiate between bacterial infection of the DU and ischemic necrosis, thus having a significant impact on clinical decision-making. The patient presented with a thermographic 'hot-spot' in the area of the DU, where the presence of deep-tissue infection was confirmed through magnetic resonance imaging and microbiological cultures. After antibiotic treatment, clinical and biochemical signs of infection subsided, but the DU did not heal. IRT showed an area of extremely low temperature in the affected toe, consis tent with critical ischemia and possibly necrosis, which mandated surgical treatment.

Another important problem in SSc is the evaluation of treatment response [19]. The action of vasodilatory agents (e.g. intravenous prostanoids and endothelin receptor antagonists) or other interventions, such as low-level laser therapy, could be potentially visualized and measured using IRT (Fig. 4). In critical digital ischemia, the effectiveness of emergency treatment such as chemical sympathectomy with lidocaine or botulinum toxin A could be immediately evaluated.

Finally, it has been suggested that following the heat challenge, a persistent temperature gradient between the fingers and the hand over $1^{\circ} \mathrm{C}$ is related to greater mortality and can be used as an independent indicator of disease severity [20].

Although promising, these potential applications are based on case reports and retrospective studies. More prospective studies on larger cohorts are needed to establish the role of IRT in SSc. The usefulness of IRT in other scleroderma-spectrum disorders, such as dermatomyositis or mixed connective tissue disease, is yet to be determined.

\section{Limitations}

Even though IRT has many advantages, there are several major limitations. Despite patient preparation, unforeseeable external and internal factors can affect skin temperature and, thereby, the outcome of the study. Moreover, there is a level of uncertainty regarding temperature measurement accuracy, due to humidity, skin emissivity and calibration of the IR camera. 

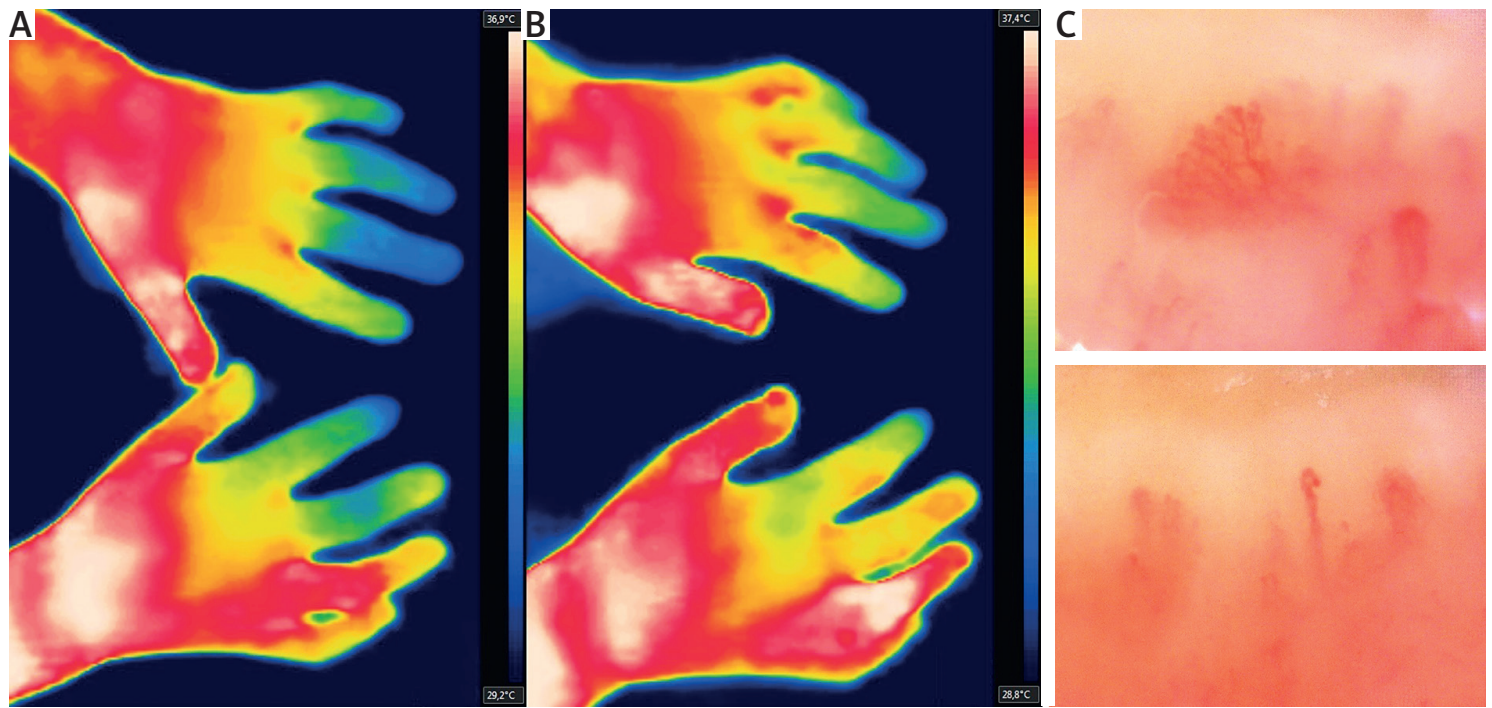

Fig. 4. Systemic sclerosis patient with digital ulcer on $4^{\text {th }}$ right finger, after low-level laser therapy. At baseline the $4^{\text {th }}$ right finger is significantly warmer $(A)$, with strong rewarming response after heat challenge, suggesting good effects of the treatment. The remaining fingers are only marginally warmer (B). Capillaroscopy consistent with 'late' scleroderma-pattern (C).

Since each centre uses different IR equipment and varying protocols, the reproducibility of the study results can be questionable and their evidence-based analysis very hard. A systemic review of the literature regarding IRT performed by Pauling et al. [21] failed to identify a single thermographic criterion that could serve as an objective endpoint in clinical trials.

Finally, IRT provides only indirect information about tissue perfusion, whereas techniques such as laser Doppler perfusion imaging (LDPI) and laser speckle contrast analysis (LASCA) allow one to actually 'see' the intensity of the blood flow in the examined area.

\section{Summary}

Despite the fact that current diagnostic standards do not recommend routine use of IRT, its potential should not be underestimated. As a first line screening tool, IRT has a role in diagnosing RP, systemic sclerosis and related connective tissue diseases, especially in conjunction with NVC. The recent introduction of low-cost IR cameras can increase the availability of IRT, which has so far been restricted to specialist tertiary care units. Such devices have a thermal resolution of $0.1^{\circ} \mathrm{C}$, which is acceptable for medical usage, and, in spite of certain limitations [22], can find a place in everyday clinical practice $[23,24]$.

The author declares no conflict of interest.

\section{References}

1. Liew SC. Electromagnetic Waves. Centre for Remote Imaging, Sensing and Processing; http://www.crisp.nus.edu.sg/ research/tutorial/em.htm.

2. Formenti D, Ludwig N, Gargano M, et al. Thermal imaging of exercise-associated skin temperature changes in trained and untrained female subjects. Ann Biomed Eng 2013; 41: 863-871.

3. Kelly G. Body temperature variability (Part 1): a review of the history of body temperature and its variability due to site selection, biological rhythms, fitness, and aging. Altern Med Rev 2006; 11: 278-293.

4. Kelly G. Body temperature variability (Part 2): masking influences of body temperature variability and a review of body temperature variability in disease. Altern Med Rev 2007; 12: 49-62.

5. Ring EFJ, Ammer K. The Technique of Infra red Imaging in Medicine. Thermol Int 2000; 10: 7-14.

6. Ring EFJ, Ammer K. Infrared thermal imaging in medicine. Physiol Meas 2012; 33: R33-46.

7. Kuryliszyn-Moskal A, Kita J, Hryniewicz A. Raynaud's phenomenon: new aspects of pathogenesis and the role of nailfold videocapillaroscopy. Reumatologia 2015; 53: 87-93.

8. Chojnowski MM, Felis-Giemza A, Olesińska M. Capillaroscopya role in modern rheumatology. Reumatologia 2016; 54: 67-72.

9. Maverakis E, Patel F, Kronenberg DG, et al. International consensus criteria for the diagnosis of Raynaud's phenomenon. J Autoimmun 2014; 48-49: 60-65.

10. Horikoshi M, Inokuma S, Kijima Y, et al. Thermal Disparity between Fingers after Cold-water Immersion of Hands: A Useful Indicator of Disturbed Peripheral Circulation in Raynaud Phenomenon Patients. Intern Med 2016; 55: 461-466.

11. Dinsdale G, Herrick A. Vascular diagnostics for Raynaud's phenomenon. J Vasc Diagn 2014; 2: 127-139. 
12. Ismail E, Orlando G, Corradini ML, et al. Differential diagnosis of Raynaud's phenomenon based on modeling of finger thermoregulation. Physiol Meas 2014; 35: 703-716.

13. Lim MJ, Kwon SR, Jung KH, et al. Digital Thermography of the Fingers and Toes in Raynaud's Phenomenon. J Korean Med Sci 2014; 29: 502-506.

14. Murray AK, Moore TL, Manning JB. et al. Noninvasive Imaging Techniques in the Assessment of Scleroderma Spectrum Disorders. Arthritis Rheum 2009; 61: 1103-1111.

15. Schlager O, Gschwandtner ME, Herberg K, et al. Correlation of infrared thermography and skin perfusion in Raynaud patients and in healthy controls. Microvasc Res 2010; 80: 54-57.

16. Cherkas LF, Carter L, Spector TD, et al. Use of thermographic criteria to identify Raynaud's phenomenon in a population setting. J Rheumatol 2003; 30: 720-722.

17. Matucci-Cerinic M, Kahaleh B, Wigley FM. Review: evidence that systemic sclerosis is a vascular disease. Arthritis Rheum 2013; 65: 1953-1962.

18. van der Weijden MA, van Vugt LM, Valk D, et al. Exploring thermography: a promising tool in differentiation between infection and ischemia of the acra in systemic sclerosis. Int I Rheum Dis 2016 Apr 2. doi: 10.1111/1756-185X.12859.
19. Kan C, Akimoto S, Abe M, et al. Preliminary thermographic evaluation of new nitroglycerine tape on the peripheral circulatory disturbance in systemic sclerosis. Ann Rheum Dis 2002; 61: 177-179.

20. Hughes M, Wilkinson J, Moore T, et al. Thermographic Abnormalities are Associated with Future Digital Ulcers and Death in Patients with Systemic Sclerosis. J Rheumatol 2016; 43: 1519-1522.

21. Pauling JD, Shipley JA, Harris ND, et al. Use of infrared thermography as an endpoint in therapeutic trials of Raynaud's phenomenon and systemic sclerosis. Clin Exp Rheumatol 2012; 30: 103-115.

22. Curran A, Klein M, Hepokoski M, et al. Improving the accuracy of infrared measurements of skin temperature. Extreme Physiol Med 2015; 4 (Suppl 1): A140.

23. Kanazawa T, Nakagami G, Goto T, et al. Use of smartphone attached mobile thermography assessing subclinical inflammation: a pilot study. J Wound Care 2016; 25: 177-180.

24. Manfredi A, Sebastiani M, Campomori F, et al. Nailfold Videocapillaroscopy Alterations in Dermatomyositis and Systemic Sclerosis: Toward Identification of a Specific Pattern. J Rheumatol 2016; 43: 1575-1580. 